associated with predation, nor with a limited supply of food. Furthermore, it was not necessarily a hunting and gathering way of life that led to altered selection pressures which were the critical factors in the emergence of active sharing behaviour in early man $^{9}$. The alternative, and equally possible, speculation is that active sharing behaviour was a part of our ancestors' repertoire long before the hunting and gathering way of life was adopted.

I thank the Director and Curators of the San Diego Zoo for facilities, and Drs I. S. Bernstein, C. R. Carpenter, C. E. Parker, D. M. Rumbaugh and $\mathrm{Mr}$ T. L. Patterson for their help. This work was supported by a University of Georgia graduate assistantship.

Michael Kavanagh

Department of Psychology,

University of Georgia,

Athens, Georgia 30601

Received June 19, 1972.

${ }^{1}$ Berkson, G., and Schusterman, R. J., Primates, 5(1-2), 1 (1964).

${ }^{2}$ Lawick-Goodall, J. van, in Primates: Studies in Adaption and Variability (edit. by Jay, P. C.), 369 (Holt, Rinehart and Winston, New York, 1968).

${ }^{3}$ Teleki, G., Sci. Amer. (in the press).

${ }^{4}$ Schaller, G., The Mountain Gorilla, 155 (University of Chicago Press, Chicago, 1963).

"Jolly, A., The Evolution of Primate Behavior, 87 (Macmillan, New York, 1972).

${ }^{6}$ Washburn, S. L., and Avis, V., in Behavior and Evolution (edit. by Roe, A., and Simpson, G. L.), 433 (Yale University Press, New Haven, 1958).

'Sahlins, M. D., in The Evolution of Man's Capacity for Culture (edit. by Spuhler, J. N.), 66 (Wayne State University Press, Detroit, 1959).

${ }^{8}$ Washburn, S. L., and Lancaster, C. S., in Human Evolution, second ed. (edit. by Korn, N., and Thompson, F.), 74 (Holt, Rinehart and Winston, New York, 1967)

${ }^{9}$ Howell, F. C., in Human Evolution, second ed. (edit. by Korn, N., and Thompson, F.), 91 (Holt, Rinehart and Winston, New York, 1967).

${ }^{10}$ Bernstein, I. S., Primates, 8, 217 (1967)

11 Bernstein, I. S., in Behavior of Nonhuman Primates (edit. by Schrier, A. M., and Stollnitz, F.), 373 (Academic Press, New York, 1971).

${ }^{12}$ Kummer, H., and Kurt, F. A., in The Baboon in Medical Research (edit. by Vagtborg, H.), 65 (University of Texas Press, Austin, 1965).

${ }^{13}$ Rowell, T. E., Anim. Behav., 15, 499 (1967).

\section{Effect of Humming on Watching Television}

RUSHTON has shown that humming causes the eyes to vibrate and this, in turn, produces a stroboscopic effect when a sectored disk is viewed in steady light ${ }^{1}$. He demonstrated this phenomenon by using a black-and-white sectored disk rotated on a phonographic turntable. When a subject hummed at the correct frequency (determined by the speed of the turntable and the number of sectors on the disk), he saw the sectors standing motionless. Slightly lower and higher pitched hums produced apparent motions of the sectors in the clockwise and counterclockwise directions, respectively.

Although unaware of Rushton's humming effect, one of us (P. C. W.) observed a related phenomenon while watching television. Humming caused the appearance of horizontal lines on the screen. The lines were visible only to the person who was humming. They could be made to stand motionless or to drift upwards or downwards on the screen, depending on the frequency of the hum. Harmonic frequencies generated multiples of the lines and very high frequencies caused a noticeable decrease in line widths.

The effect is most clearly seen if the subject stands well away from the set. This would seem to support Rushton's idea that the effect is due to the displacement of the image on the retina because the effect of small displacements caused by vibration would be more enhanced the smaller the visual angle being subtended.

The stripes show up as grey and indistinct areas on both black-and-white and colour sets. Apparently some visual information is being encoded for; if it were not, the stripes would probably look black.

We hope that by describing this effect we will help bring Rushton's humming phenomenon within easy test of those who do not have phonographs but who do occasionally watch television.

Philip C. Williams

3105 Ortega Drive,

Tallahassee, Florida 32303

Theodore P. Williams

Institute of Molecular Biophysics,

Florida State University,

Tallahassee, Florida 32306

Received July 10, 1972.

${ }^{1}$ Rushton, W. A. H., Nature, 216, 1173 (1967).

\section{The Fourier Transform of Evoked Responses}

INVESTIGATIONS into the relationship between evoked potentials and human intelligence have been carried out by $\mathrm{Ertl}^{1}$, who claims to have found no evidence to support my earlier conclusion $^{2}$ regarding this relationship. In my opinion, Ertl's work supports my conclusion that there is a significant difference between the evoked potentials for high and low intelligence groups. Although Ertl uses a Fourier analysis rather different from my own analysis, it should be possible to relate the two investigations (see, for example, ref. 3 ).

I find it necessary to show this relationship by prediction from the results given in my letter because of the difficulties encountered in the frequency domain. According to my work, Ertl's group of high IQ subjects would show a natural resonant frequency of $14 \mathrm{~Hz}$ or more, and his low IQ group a frequency of $6 \mathrm{~Hz}$ or less. Because the frequencies mentioned in ref. 2 are natural resonant frequencies, it is necessary to multiply them by a factor $\frac{1}{\sqrt{1-\zeta^{2}}}$ to shift these frequencies to the axis along which Ertl has evaluated the transform ${ }^{4}$. My records indicate a considerable variation in the value of the damping factor $\zeta$, but a value of 0.7 would seem to be about average. On performing the necessary calculations, I would expect the apparent frequencies in the Fourier transform to be $19.6 \mathrm{~Hz}$ or more, and $8.4 \mathrm{~Hz}$ or less. In Fig. 1 (ref. 1), the curve for the high IQ subjects has a peak above the characteristic shape of the transform at $20 \mathrm{~Hz}$ and the curve for the low IQ subjects has a peak above the characteristic shape at $8 \mathrm{~Hz}$. Fig. 2 also shows the same frequency components, but the accuracy is lower because of the shorter evaluation period.

It would therefore seem that Ertl's investigation tends to confirm my original findings, rather than disproving them.

Capilano College,

West Vancouver,

British Columbia

Received July 26, 1971; final revision July 19, 1972. 